\title{
Paths and cycles in colored graphs*
}

\author{
HAJo Broersma \\ Department of Applied Mathematics \\ University of Twente, P. O. Box 217, 7500 AE Enschede \\ The Netherlands \\ Xueliang Li \\ Center for Combinatorics \\ Nankai University, Tianjin $3000 \% 1$ \\ P.R. China \\ Gerhard Woeginger \\ Department of Applied Mathematics \\ University of Twente, P. O. Box 217, 7500 AE Enschede \\ The Netherlands
}

\section{SHENGGUi ZHANG}

Department of Applied Mathematics

Northwestern Polytechnical University, Xi'an, Shaanxi 710072

P.R. China

\begin{abstract}
Let $G$ be an (edge-)colored graph. A path (cycle) is called monochromatic if all of its edges have the same color, and is called heterochromatic if all of its edges have different colors. In this paper, some sufficient conditions for the existence of (long) monochromatic paths and cycles, and those for the existences of long heterochromatic paths and cycles are obtained. It is proved that the problem of finding a path (cycle) with as few different colors as possible in a colored graph is NP-hard. Exact and approximation algorithms for finding a path with the fewest colors are provided. The complexity of the exact algorithm and the performance ratio of the approximation algorithm are analyzed. We also pose a problem on the existence of paths and cycles with many different colors.
\end{abstract}

* Supported by NSFC. 


\section{Introduction}

We use Bondy and Murty [3] for terminology and notations not defined here and consider simple graphs only.

Let $G=(V, E)$ be a graph. By an edge-coloring of $G$ we will mean a function $C: E \rightarrow \mathbb{N}$, the set of nonnegative integers. If $G$ is assigned such a coloring, then we say that $G$ is a colored graph, denote the colored graph by $(G, C)$, and call $C(e)$ the color of the edge $e \in E$. All edges with the same color form a color class of the graph. We note that $C$ is not necessarily a proper edge-coloring, i.e., two adjacent edges may have the same color. For a subgraph $H$ of $G$, we let $C(H)=\cup_{e \in E(H)}\{C(e)\}$ and $c(H)=|C(H)|$. For a vertex $v$ of $G$, the color neighborhood $C N(v)$ of $v$ is defined as the set $\{C(e): e$ is incident with $v\}$ and the color degree $d^{c}(v)=|C N(v)|$. A path (cycle) is called monochromatic if all of its edges have the same color; and it is called heterochromatic if all of its edges have different colors.

If we regard an uncolored graph as a colored graph in which all edges have different colors, then the number of colors of a subgraph is simply the number of its edges, and the color degree of a vertex is the degree of it.

There are many existing literature dealing with the existence of paths and cycles with special properties in edge-colored graphs. In [19], the authors showed that for every positive integer $t$, every real number $\delta, 0<\delta<\frac{1}{2 t-1}$, and every $n$ sufficiently large with respect to $t$ and $\delta$, there exists a graph $G$ on $n$ vertices, such that $G$ has girth at least $t+2$ and every proper edge coloring of $G$ contains a heterochromatic cycle of length $i$, for all $i, 2 t+2 \leq i \leq n^{\delta}$. In [5], the authors showed that for a 2-edge-colored graph $G$ and three specified vertices $x, y, z$, to decide whether there exists a color-alternating path from $x$ to $y$ passing through $z$ is NP-complete. Many results deal with colored complete graphs. In [13] Giraud studied the existence of monochromatic triangles and heterochromatic triangles in colored complete graph. A problem on the conditions for a colored complete graph to contain heterochromatic Hamiltonian cycles was mentioned in [8] by Erdös, Nešetřil and Rödl. The heterochromatic Hamiltonian cycle or path problem was also studied by Hahn and Thomassen [14], Rödl and Winkler (see [11]), Frieze and Reed [11], and Albert, Frieze and Reed [1]. For more references, see $[2,9,10,16,17]$. Many results in these papers are proved by using probabilistic methods.

This paper contains some basic results on paths and cycles in general colored graphs. In Sections 2 and 3, we give some sufficient conditions for the existence of (long) monochromatic paths and cycles, and those for the existence of long heterochromatic paths and cycles. In Section 4, we prove that the problem of finding a path (cycle) with as few different colors as possible between two given vertices in a colored graph is NP-hard and propose two exact algorithms and two approximation algorithms for finding a path with the fewest colors. The complexity of the two exact algorithms and the performance ratio of the approximation algorithms are analyzed. We also pose a problem on the existence of paths and cycles with many different colors in Section 5. 


\section{Monochromatic paths and cycles}

First, let us consider the problem under what conditions a colored graph contains a monochromatic path or a monochromatic cycle. It is clear that every colored graph contains at least one monochromatic path. Moreover, it is obvious that not every colored graph contains monochromatic cycles.

The arboricity $a(G)$ of a graph $G$ is defined as the minimum number of edgedisjoint forests into which $G$ can be decomposed. Clearly, it is also the minimum number of colors necessary to color the edges of $G$ so that no cycle is monochromatic. So we have

Remark 1 Let $G$ be a colored graph. If $c(G)<a(G)$, then $G$ contains at least one monochromatic cycle.

The arboricity $a(G)$ can be determined by applying the matroid partitioning algorithm of Edmonds [15]. In [18] Picard and Queyranne showed that this parameter can be determined in at most $O\left(n^{4}\right)$ operations, by using network flow methods. It is (almost) trivial to check whether a colored graph contains a monochromatic cycle: for each color class $E_{i}$ check whether the induced subgraph $G\left[E_{i}\right]$ contains a cycle.

The following result on the existence of monochromatic paths and cycles with a prescribed length is obvious.

Remark 2 Let $G$ be a colored graph with color classes $E_{1}, E_{2}, \ldots, E_{c}$. Then $G$ has a monochromatic path (cycle) of length $l$ if and only if for some $i$ with $1 \leq i \leq c$, the induced subgraph $G\left[E_{i}\right]$ has a path (cycle) of length $l$.

If we regard an uncolored graph $G$ as a colored graph $(G, C)$ for which all edges have the same color, then $(G, C)$ contains a monochromatic path (cycle) of length at least $l$ if and only if $G$ contains a path (cycle) of length at least $l$. Since the problem of deciding whether for general $l$ there is a path (cycle) of length at least $l$ in an (uncolored) graph is NP-complete, the problem of deciding whether there is a monochromatic path (cycle) of length at least $l$ in a colored graph is also NPcomplete.

There are many results on the existence of long paths and cycles in (uncolored) graphs. Here we list two of them.

Theorem A (Erdös and Gallai [7]) Let $G$ be a graph of order $n$ and size $m$. Then $G$ contains a path of length at least $\frac{2 m}{n}$.

Theorem B (Erdös and Gallai [7]) Let $G$ be a graph of order $n$ and size $m$ such that $m \geq n$. Then $G$ contains a cycle of length at least $\frac{2 m}{n-1}$.

Using Remark 2 and Theorems A and B, it is not difficult to prove the following results:

Proposition 3 Suppose that $G$ is a colored graph of order $n$ and size $m$. Then $G$ contains a monochromatic path of length at least $\frac{2 m}{c(G) n}$. 
Proposition 4 Suppose that $G$ is a colored graph of order $n$ and size $m$ such that $m \geq c(G) n$. Then $G$ contains a monochromatic cycle of length at least $\frac{2 m}{c(G)(n-1)}$.

As it was shown in [7], both Theorems A and B are best possible. To see the sharpness of Theorem A, let $p K_{r}$ denote the disjoint union of $p$ copies of $K_{r}$. This graph has $n=p r$ vertices and $m=\frac{p r(r-1)}{2}$ edges. It is easy to check that $\frac{2 m}{n}=r-1$. On the other hand, $p K_{r}$ contains no path of length greater than $r-1$. Of course, this graph also shows that the result in Proposition 3 in the case $c(G)=1$ is best possible. This example can be extended to general cases to show the sharpness of the result in Proposition 3.

Let $G$ and $H$ be two colored graphs. The colored Cartesian product of $G$ and $H$ is the graph $G \times H$ with a coloring defined as follows: From the definition of the Cartesian product of graphs, to every vertex $u$ of $G$, there corresponds a subgraph $H_{u}$ of $G \times H$ such that $H_{u}$ is isomorphic to $H$. To each edge $e$ of $H_{u}$, assign the color of the edge corresponding to $e$ in $H$. Similarly, to every vertex $v$ of $H$, there corresponds a subgraph $G_{v}$ of $G \times H$ such that $G_{v}$ is isomorphic to $G$. To each edge $e$ of $G_{v}$, assign the color of the edge corresponding to $e$ in $G$. The colored Cartesian product $G_{1} \times G_{2} \times \cdots \times G_{k}$ of $k \geq 2$ colored graphs $G_{1}, G_{2} \cdots, G_{k}$ can be defined inductively.

Let $G_{i}(1 \leq i \leq c)$ be the colored graph $K_{r}$ such that all the edges of $G_{i}$ receive the same color $i$. By $K_{r}^{c}$ we denote the colored Cartesian product $G_{1} \times G_{2} \times \cdots \times G_{c}$. It is not difficult to see that the colored graph $K_{r}^{c}$ has $n=r^{c}$ vertices, $m=\frac{c r^{c}(r-1)}{2}$ edges and $c$ colors. This implies that $\frac{2 m}{c n}=r-1$. On the other hand, the colored graph $K_{r}^{c}$ has no monochromatic path of length greater than $r-1$. This shows that the result in Proposition 3 is best possible. Clearly the disjoint union of some copies of the colored graph $K_{r}^{c}$ defined above can also be used to show the sharpness of the result of Proposition 3.

The sharpness of Theorem B can be shown by the graph $\Gamma_{p, r}$ defined as follows: The graph $\Gamma_{p, r}$ is a connected graph which has exactly $n=p(r-1)+1$ vertices and each of the $p$ blocks of it is a clique on $r$ vertices. This graph has $m=\frac{p r(r-1)}{2}$ edges and clearly $\frac{2 m}{n-1}=r$. On the other hand, it has no cycle of length greater than $r$. Of course this example also shows that the result of Proposition 4 is best possible in the case $c(G)=1$.

Let $G_{p_{i}}(1 \leq i \leq c)$ be the colored graph $\Gamma_{p_{i}, r}$ such that all the edges of it receive the same color $i$. Denote by $G$ the colored Cartesian product $G_{p_{1}} \times G_{p_{2}} \times \cdots \times G_{p_{c}}$. Then $G$ has

$$
n=\sum_{i=1}^{c} \sum_{1 \leq j_{1}<j_{2}<\cdots<j_{i} \leq c} p_{j_{1}} p_{j_{2}} \cdots p_{j_{i}}(r-1)^{i}+1
$$


vertices,

$$
m=\frac{r(r-1)}{2} \sum_{i=1}^{c} i \sum_{1 \leq j_{1}<j_{2}<\cdots<j_{i} \leq c} p_{j_{1}} p_{j_{2}} \cdots p_{j_{i}}(r-1)^{i-1}
$$

edges, and $c$ colors. Therefore,

$$
\begin{aligned}
\frac{2 m}{c(n-1)} & =\frac{2 \times \frac{r(r-1)}{2} \sum_{i=1}^{c} i \sum_{1 \leq j_{1}<j_{2}<\cdots<j_{i} \leq c} p_{j_{1}} p_{j_{2}} \cdots p_{j_{i}}(r-1)^{i-1}}{c\left(\sum_{i=1}^{c} \sum_{1 \leq j_{1}<j_{2}<\cdots<j_{i} \leq c} p_{j_{1}} p_{j_{2}} \cdots p_{j_{i}}(r-1)^{i}+1-1\right)} \\
& =r-\frac{\sum_{i=1}^{c-1}(c-i) \sum_{1 \leq j_{1}<j_{2}<\cdots<j_{i} \leq c} p_{j_{1}} p_{j_{2}} \cdots p_{j_{i}}(r-1)^{i-1}}{c \sum_{i=1}^{c} \sum_{1 \leq j_{1}<j_{2}<\cdots<j_{i} \leq c} p_{j_{1}} p_{j_{2}} \cdots p_{j_{i}}(r-1)^{i-1}} .
\end{aligned}
$$

It is clear that $\left\lceil\frac{2 m}{c(n-1)}\right\rceil=r$. On the other hand, the colored graph $G$ contains no monochromatic cycle of length greater than $r$. This shows that the result in Proposition 4 is best possible.

\section{Heterochromatic paths and cycles}

If we regard an uncolored graph $G$ as a colored graph $(G, C)$ in which all edges have different colors, then for general $l G$ contains a path (cycle) of length at least $l$ if and only if $(G, C)$ contains a heterochromatic path (cycle) of length at least $l$. As we mentioned earlier, the problem of deciding whether there is a path (cycle) of length at least $l$ in an (uncolored) graph is NP-complete. Therefore the problem of deciding whether there is a heterochromatic path (cycle) of length at least $l$ in a colored graph is $\mathbf{N P}$-complete, too. In this section we will consider under what conditions there is a heterochromatic path (cycle) with a prescribed length in a colored graph.

Let $G$ be a colored graph. By selecting precisely one edge from each color class of $G$, we obtain a new colored graph $G^{\prime}$, such that all the edges of $G^{\prime}$ have different colors, and $c\left(G^{\prime}\right)=c(G)$. Using Theorems A and B, it is easy to prove the following results.

Proposition 5 Let $G$ be a colored graph of order $n$. Then $G$ contains a heterochromatic path of length at least $\frac{2 c(G)}{n}$.

Proposition 6 Let $G$ be a colored graph of order $n$ such that $c(G) \geq n$. Then $G$ contains a heterochromatic cycle of length at least $\frac{2 c(G)}{n-1}$.

Furthermore, we have the following two results on the existence of long heterochromatic paths. 
Proposition 7 Let $G$ be a colored graph and $k$ an integer. Suppose that $d^{c}(v) \geq k$ for every vertex $v$ of $G$. Then for every vertex $z$ of $G$ there exists a heterochromatic path of length at least $\left\lceil\frac{k+1}{2}\right\rceil$ with $z$ as one of its end vertices.

Proof Choose a longest heterochromatic $z$-path $P$ with length $l$. Denote the other end-vertex of $P$ as $v$. Then from the assumption of the proposition, we know that all incident edges of $v$ with the other end not on $P$ have colors also appearing in $E(P)$. Therefore, $d^{c}(v) \leq l+(l-1)=2 l-1$. On the other hand, $d^{c}(v) \geq k$, so we have $l \geq\left\lceil\frac{k+1}{2}\right\rceil$.

If one considers heterochromatic paths which do not need to start from a given vertex $z$, A. Saito showed that $l \geq\left\lceil\frac{k+1}{2}\right\rceil+1$, and furthermore, he conjectured that $l \geq\left\lceil\frac{2 k+1}{3}\right\rceil$.

Proposition 8 Let $G$ be a colored graph and $s$ an integer. Suppose that $\mid C N(u) \cup$ $C N(v) \mid \geq s>1$ for every pair of vertices $u$ and $v$ of $G$. Then $G$ contains a heterochromatic path of length at least $\left\lceil\frac{s}{3}\right\rceil+1$.

Proof Choose a longest heterochromatic path $P$ with length $l$. Denote the endvertices of $P$ as $u$ and $v$. Then from the assumption of the proposition, we know that all incident edges of $u$ and $v$ with the other end not on $P$ have colors also appearing in $E(P)$. Therefore, $|C N(u) \cup C N(v)| \leq l+(l-1)+(l-2)=3 l-3$. On the other hand, $|C N(u) \cup C N(v)| \geq s$, so we have $l \geq\left\lceil\frac{s}{3}\right\rceil+1$.

In the following, we give a sufficient condition for the existence of heterochromatic triangles or quadrilaterals.

Proposition 9 Let $G$ be a colored graph of order $n \geq 4$, such that $\mid C N(u) \cup$ $C N(v) \mid \geq n-1$ for every pair of vertices $u$ and $v$ of $G$. Then $G$ contains at least one heterochromatic triangle or one heterochromatic quadrilateral.

Proof If $|C N(u)|=n-1$ for every vertex $u$ of $G$, then $d(u)=n-1$, and $G$ is a complete graph. It is clear that every triangle of $G$ is heterochromatic. So, we need only consider the case that there is some vertex $u \in V(G)$ with $|C N(u)|<n-1$.

Suppose that $G$ contains neither heterochromatic triangles nor heterochromatic quadrilaterals. Without loss of generality, we can assume that $V(G)=\left\{x_{1}, x_{2}, \ldots\right.$, $\left.x_{k}, u, v, y_{k+2}, y_{k+3}, \ldots, y_{n-2}, y_{n-1}\right\}, d^{c}(u)=k+1<n-1, C\left(u x_{i}\right)=i$ for $i=$ $1,2, \ldots, k, C(u v)=k+1$ and $C\left(v y_{j}\right)=j$ for $j=k+2, \ldots, n-1$.

First, consider the vertex $u$ and a vertex $x_{i} \in\left\{x_{1}, x_{2}, \ldots, x_{k}\right\}$. Since $C N(v) \cup$ $\left(C N\left(x_{i}\right) \cap\left\{C\left(x_{i} x_{1}\right), C\left(x_{i} x_{2}\right), \ldots, C\left(x_{i} x_{i-1}\right), C\left(x_{i} x_{i+1}\right), \ldots, C\left(x_{i} x_{k}\right), C\left(x_{i} u\right)\right\}\right) \subseteq$ $\{1,2, \ldots, k+1\}$ and $\left|C N(u) \cup C N\left(x_{i}\right)\right| \geq n-1, x_{i}$ must be adjacent to each vertex $y_{j} \in\left\{y_{k+2}, y_{k+3}, \ldots, y_{n-1}\right\}$, and $C\left(x_{i} y_{j}\right)=j$ by our assumption that $G$ contains neither heterochromatic triangles nor heterochromatic quadrilaterals.

Now consider the two vertices $u$ and $y_{n-1}$. Since $C N(u) \cup\left(C N\left(y_{n-1}\right) \cap\left\{C\left(y_{n-1} x_{1}\right)\right.\right.$, $\left.\left.C\left(y_{n-1} x_{2}\right), \ldots, C\left(y_{n-1} x_{k}\right)\right\}\right) \subseteq\{1,2, \ldots, k+1, n-1\}$ and $\left|C N(u) \cup C N\left(y_{n-1}\right)\right| \geq n-1$, 
we have that $y_{n-1} y_{j} \in E(G)$ and $C\left(y_{n-1} y_{j}\right)=j$ for $j=k+2, \ldots, n-2$ by our assumption that $G$ contains no heterochromatic triangles.

So, we have $C N(u) \cup\left(C N\left(y_{n-2}\right) \cap\left\{C\left(y_{n-2} x_{1}\right), C\left(y_{n-2} x_{2}\right), \ldots, C\left(y_{n-2} x_{k}\right)\right.\right.$, $\left.\left.C\left(y_{n-2} u\right), C\left(y_{n-2} v\right), C\left(y_{n-2} y_{n-1}\right)\right\}\right) \subseteq\{1,2, \ldots, k, k+1, n-2\}$. Therefore, $\mid C N(u)$ $\cup C N\left(y_{n-2}\right)|\leq|\{1,2, \ldots, k, k+1, n-2\}|+|\left\{y_{k+2}, y_{n+3}, \ldots, y_{n-3}\right\} \mid=(k+2)+(n-$ $k-4)=n-2<n-1$, a contradiction.

The proof of the result is complete.

Although the proofs of the results in Propositions 7 to 9 are easy, it can be shown that these results are best possible in the sense that there exist some graphs of small orders showing that they cannot be improved. However, we think that perhaps much stronger results are possible to obtain if one excludes some small counter-examples or simple classes of counter-examples. The proof techniques we applied here do not seem to be strong enough for obtaining such improvements. Maybe an approach using probabilistic proof techniques could yield such improvements.

\section{Paths and cycles with few colors}

If we regard an (uncolored) graph $G$ as a colored graph $(G, C)$ for which all edges have different colors, then a shortest path between two given vertices in $G$ is a path between the two vertices with the fewest colors in the colored graph $(G, C)$. It is well-known that the problem of finding a shortest path between two given vertices in a (weighted) graph can be solved efficiently. There are many polynomial-time algorithms to solve this problem. In this section, we will consider the complexity aspects of finding a path between two given vertices with the fewest colors in a colored graph.

\section{Problem 10}

INSTANCE: Graph $G=(V, E)$ with a coloring $C: E \rightarrow \mathbb{N}$ and two given vertices $s_{0}$ and $t_{0}$, positive integer $K \leq c(G)$.

QUESTION: Is there a path $P$ from $s_{0}$ to $t_{0}$ such that $c(P) \leq K$ ?

The following problem is NP-complete, see [12] for a reference.

\section{3-SATISFIABILITY (3-SAT)}

INSTANCE: Collection $C=\left\{C_{1}, C_{2}, \ldots, C_{m}\right\}$ of clauses on a finite set $U$ of variables such that $\left|C_{i}\right|=3$ for $1 \leq i \leq m$.

QUESTION: Is there a truth assignment for $U$ that satisfies all the clauses in $C$ ?

In this section, we use this result to show that Problem 10 is NP-complete, too.

Theorem 11 Problem 10 is NP-complete.

Proof It is easy to see that Problem 10 is in NP. One way to see this is to observe that a nondeterministic algorithm need only guess an $\left(s_{0}, t_{0}\right)$-path $P$ in $G$, and check in linear time whether $c(P) \leq K$.

We shall now show that 3-SAT can be polynomially transformed to Problem 10 . 
Given a Boolean formula $F$ consisting of $m$ clauses $C_{1}, C_{2}, \ldots, C_{m}$ (with three literals per clause) and involving $n$ variables $x_{1}, x_{2}, \ldots, x_{n}$, we shall construct a graph $G=$ $(V, E)$ with a coloring $C: E \rightarrow \mathbb{N}$ and two vertices $s_{0}$ and $t_{0}$, such that $G$ has an $\left(s_{0}, t_{0}\right)$-path $P$ with $c(P) \leq n+1$ if and only if $F$ is satisfiable.

First, for the variable $x_{i}(1 \leq i \leq n)$, we construct a subgraph $A_{i}$ of $G$, where $V\left(A_{i}\right)=\left\{s_{i-1}, u_{i 1}, u_{i 2}, s_{i}\right\}$ and $\bar{E}\left(A_{i}\right)=\left\{s_{i-1} u_{i 1}, u_{i 1} s_{i}, s_{i-1} u_{i 2}, u_{i 2} s_{i}\right\}$. Assign a special color 0 to the edges $s_{i-1} u_{i 1}$ and $s_{i-1} u_{i 2}$, the color $i$ to the edge $u_{i 1} s_{i}$ and the color $i^{\prime}$ to the edge $u_{i 2} s_{i}$ for $i=1,2, \ldots, n$. Then we get a colored graph $A=\cup_{i=1}^{n} A_{i}$. For the clause $C_{j}(1 \leq j \leq m)$, we construct a subgraph $B_{j}$ of $G$, where $V\left(B_{j}\right)=$ $\left\{t_{j-1}, v_{j 1}, v_{j 2}, v_{j 3}, t_{j}\right\}$ and $E\left(B_{j}\right)=\left\{t_{j-1} v_{j 1}, t_{j-1} v_{j 2}, t_{j-1} v_{j 3}, v_{j 1} t_{j}, v_{j 2} t_{j}, v_{j 3} t_{j}\right\}$. For $j=1,2, \ldots, m$ and $k=1,2,3$, assign the color 0 to the edge $t_{j-1} v_{j k}$, the color $h$ to the edge $v_{j k} t_{j}$ if the $k$ th literal of $C_{j}$ is $x_{h}$ and the color $h^{\prime}$ to the edge $v_{j k} t_{j}$ if the $k$ th literal of $C_{j}$ is $\bar{x}_{h}$. Then we get a colored graph $B=\cup_{j=1}^{m} B_{j}$. The colored graph $G$ is obtained by connecting the two graphs $A$ and $B$ with an edge $s_{n} t_{m}$ and coloring this edge with the color 0 , see Figure 1 . Clearly the construction of $G$ can be accomplished in polynomial time.



Figure 1: The graph in the proof of Theorem 11 in the case $C_{2}=x_{1} \bar{x}_{2} x_{3}$.

It is not difficult to verify that there is an $\left(s_{0}, t_{0}\right)$-path $P$ with $c(P) \leq n+1$ if and only if $F$ is satisfiable. We leave the details to the reader.

The following consequence of Theorem 11 is immediate.

Corollary 12 For a given pair of vertices $s$ and $t$ in a colored graph $G$, finding a path between $s$ and $t$ in $G$ with as few different colors as possible is NP-hard.

Remark 13 Broersma and $\mathrm{Li}$ [4] proved that the problem of finding a spanning tree with as few colors as possible in a colored graph is NP-hard by using the minimum dominating set problem. It is not difficult to see that the graph $G$ we constructed in the proof of Theorem 11 has a spanning tree with at most $n+1$ colors if and only if 
$F$ is satisfiable. So, our technique also provides a new proof to Broersma and Li's result.

The problem of finding a cycle with as few colors as possible in a colored graph is also NP-hard. We consider the following decision problem.

\section{Problem 14}

INSTANCE: 2-connected graph $G=(V, E)$ with a coloring $C: E \rightarrow \mathbb{N}$ and a given vertex $u$, positive integer $K \leq c(G)$.

QUESTION: Is there a cycle $C$ passing through $u$ such that $c(C) \leq K$ ?

Theorem 15 Problem 14 is NP-complete.

Proof Let $G$ a connected colored graph. Construct a 2-connected colored graph $G^{\prime}$ by adding a new vertex $u$, connecting $u$ to every vertex $v$ of $G$ with an edge and assigning an extra color 0 to the new edges. Then $G$ contains a path $P$ such that $c(P) \leq K$ if and only if $G^{\prime}$ contains a cycle $C$ passing through $u$ such that $c(C) \leq K+1$. It follows from Corollary 12 that Problem 14 is $\mathbf{N P}$-complete.

Corollary 16 Finding a cycle with as few colors as possible in a 2-connected colored graph is NP-hard.

As we proved in Theorem 11, finding a path with as few colors as possible (minimum path) between two given vertices in a colored graph is NP-hard. However, if $c(G)$ is much smaller than $|V(G)|$, say, $c(G)=O\left(\log _{2}|V(G)|\right)$, there will be some efficient algorithm for solving this problem.

An approach to finding a minimum path between two given vertices $s_{0}$ and $t_{0}$ is to check whether there is an $\left(s_{0}, t_{0}\right)$-path in the graphs $G\left[E_{i_{1}}\right]$ with $1 \leq i_{1} \leq k$, $G\left[E_{i_{1}} \cup E_{i_{2}}\right]$ with $1 \leq i_{1}, i_{2} \leq k$ and $i_{1} \neq i_{2}, \ldots$, and $G\left[E_{i_{1}} \cup E_{i_{2}} \cup \cdots \cup E_{i_{d}}\right]$ with $1 \leq i_{1}, i_{2}, \ldots, i_{d} \leq k$ and $i_{p} \neq i_{q}$ for $1 \leq p \neq q \leq k$, where $E_{1}, E_{2}, \ldots, E_{k}$ are the color classes, $k=c(G)$, and $d$ is the distance between $s_{0}$ and $t_{0}$. The complexity of such an algorithm is

$$
\begin{aligned}
& {\left[\left(\begin{array}{l}
k \\
1
\end{array}\right)+\left(\begin{array}{l}
k \\
2
\end{array}\right)+\cdots+\left(\begin{array}{c}
k \\
\min \{d, k\}
\end{array}\right)\right] O(|V(G)|)} \\
& =\min \left\{O\left(k^{d}|V(G)|\right), O\left(2^{k}|V(G)|\right)\right\} .
\end{aligned}
$$

It is of interest to consider approximation algorithms for the minimum path problem. If we use a shortest path between two vertices as an approximate solution for a minimum path, the approximation ratio is $c(G)$. We can also design an approximation algorithm which is similar to Dijkstra's Algorithm for finding a shortest path.

\section{Algorithm 17}

Step 1. Set $C\left(s_{0}\right)=\emptyset, C\left(S_{0}\right)=0, c(v)=\infty$ for $v \neq s_{0}, S_{0}=\left\{s_{0}\right\}$ and $i=0$.

Step 2. For each $v \in V \backslash S_{i}$, replace $C(v)$ by $C\left(u_{i}\right) \cup\left\{C\left(u_{i} v\right)\right\}$ if $c(v)>\mid C\left(u_{i}\right) \cup$ $\left\{C\left(u_{i} v\right)\right\} \mid$ and set $c(v)=|C(v)|$. Compute $\min _{v \in V \backslash S_{i}}\{c(v)\}$ and let $u_{i+1}$ denote a vertex for which this minimum is attained. Set $S_{i+1}=S_{i} \cup$ $\left\{u_{i+1}\right\}$. 
Step 3. If $u_{i+1}=t_{0}$, stop. Otherwise, replace $i$ by $i+1$ and go to Step 2 .

The approximation factor of this algorithm can get arbitrarily large. This can be shown by the graph in Figure 2.



Figure 2

The graph in Figure 2 is constructed as follows. Let $H_{0}$ be an $\left(s_{0}, t_{0}\right)$-path $P_{0}$ with $m+n$ edges and assign the colors $1^{(0)}, 2^{(0)}, \ldots,(m+n)^{(0)}$ to the edges of $P_{0}$, respectively. For $1 \leq i \leq m$, the graph $H_{i}$ is obtained from $H_{i-1}$ by adding a new $\left(s_{i-1}, t_{i-1}\right)$-path $P_{i}$ with $m+n-i$ edges and assigning the colors $1^{(i)}, 2^{(i)}, \ldots,(m+$ $n-i)^{(i)}$ to the edges of $P_{i}$, respectively. We denote the two vertices $s_{i-1}$ and $t_{i-1}$ of $H_{i}$ by $s_{i}$ and $t_{i}$, respectively. By denoting the vertices $s_{i}$ by $s_{i}^{\prime}$ and $t_{i}$ by $t_{i}^{\prime}$, we get a new graph $H_{i}^{\prime}$ for each $i$ with $1 \leq i \leq m$. The graph in Figure 2 is constructed from the graphs $H_{0}, H_{i}$ and $H_{i}^{\prime}(1 \leq i \leq m)$ by identifying the vertices $t_{0}$ with $s_{1}^{\prime}$, the vertices $s_{i-1}$ with $t_{i}$, and the vertices $s_{i}^{\prime}$ with $t_{i-1}^{\prime}$ for $1 \leq i \leq m$.

It is easy to see that the minimum path between $s_{m}$ and $t_{m}^{\prime}$ in the graph $H$ is of $m+n$ colors. Whereas we will get an approximate result $n(m+1)+\frac{m(m+1)}{2}$ if we apply the above algorithm to the graph $H$. So the approximation factor is

$$
\left|1-\frac{n(m+1)+\frac{m(m+1)}{2}}{m+n}\right|=\frac{m n+\frac{m(m+1)}{2}}{m+n} \rightarrow \infty
$$

when $m \rightarrow \infty$ in the case $n=1$ or $n=m^{1+\epsilon}(\epsilon>0)$. 


\section{$5 \quad$ Paths and cycles with many colors}

If we regard an uncolored graph as a colored graph in which all edges have different colors, then the number of colors of a subgraph is just the number of edges of it. It is well-known that the problem of finding a longest path or a longest cycle in a graph is NP-hard. Therefore, the problem of finding a path or a cycle with as many colors as possible in a colored graph are also NP-hard.

In the past decades, many sufficient conditions for the existence of long paths and cycles have been derived. The oldest result of this type is due to Dirac.

Theorem C (Dirac [6]) Let $G$ be graph and $d$ an integer. If $d(v) \geq d$ for every vertex $v$ of $G$, then $G$ contains (1) a path of length at least d, and (2) a cycle of length at least $d+1$ if $d>1$.

It is an interesting problem to establish whether Theorem $\mathrm{C}$ admits a generalization to colored graphs. This leads to the following problem.

Problem 18 Let $G$ be a colored graph such that $d^{c}(v) \geq d$ for every vertex $v$ of $G$, where $d$ is an nonnegative integer. For what values $p$ and $c$ does $G$ contain a path with at least $p$ colors, and a cycle with at least $c$ colors if $d>1$ ?

There are some examples which show that in Problem 18, both $p$ and $c$ cannot be greater than $d-1$.

By imposing a higher connectivity, the bound on the cycle length in Theorem $\mathrm{C}$ can be increased.

Theorem D (Dirac [6]) Let $G$ be a 2-connected graph and $d$ an integer. If $d(v) \geq d$ for every vertex $v$ of $G$, then $G$ contains either a Hamilton cycle or a cycle of length at least $2 d$.

Let $K_{n, n+1}$ be the complete bipartite graph with bipartition $(X, Y)$ such that $|X|=n$ and $|Y|=n+1$. Assign a coloring to $K_{n, n+1}$ as follows: first color the graph $K_{n, n+1}-y$ for some vertex $y \in Y$ by a proper $n$-edge-coloring, then assign the same $n$ colors of $K_{n, n+1}$ to the $n$ edges incident to $y$, respectively. It is easy to show that $d^{c}(v) \geq n$ for each vertex $v$ of $K_{n, n+1}$, but $K_{n, n+1}$ contains neither a Hamilton cycle nor a cycle with more than $n$ colors. This shows that, different from Theorem $\mathrm{D}$, imposing a higher connectivity on the graphs in Conjecture 18 cannot guarantee the existence of cycles with more colors.

Acknowledgement: The authors are grateful to the referees for their suggestions and comments, which are very helpful for improving the presentation of this paper.

\section{References}

[1] M. Albert, A. Frieze and B. Reed, Multicolored Hamilton cycles, Electronic Journal of Combinatorics $\mathbf{2}$ (1995), \#R10.

[2] M. Axenovich, T. Jiang and Zs. Tuza, Local anti-Ramsey numbers of graphs, Combin. Probab. Comput. 12 (2003), 495-511. 
[3] J.A. Bondy and U.S.R. Murty, Graph Theory with Applications, Macmillan London and Elsevier, New York (1976).

[4] H.J. Broersma and X. Li, Spanning trees with many or few colors in edge-colored graphs, Discuss. Math. Graph Theory 17 (1997), 259-269.

[5] W.S. Chou, Y. Manoussakis, O. Megalalaki, M. Spyratos and Zs. Tuza, Paths through fixed vertices in edge-colored graphs, Math. Inf. Sci. Hum. 32 (1994), 49-58.

[6] G.A. Dirac, Some theorems on abstract graphs, Proc. London Math. Soc. 2 (1952), no. 3, 69-81.

[7] P. Erdös and T. Gallai, On maximal paths and circuits in graphs, Acta Math. Sci. Hung. 10 (1959), 337-356.

[8] P. Erdös, J. Nešetřil and V. Rödl, Some problems related to partitions of edges of a graph, in Graphs and Other Combinatorial Topics, Teubner, Leipzig (1983), 54-63.

[9] P. Erdös and Zs. Tuza, Rainbow Hamiltonian paths and canonically colored subgraphs in infinite complete graphs, Mathematica Pannonica 1 (1990), 5-13.

[10] P. Erdös and Tuza, Rainbow subgraphs in edge-colorings of complete graphs, Ann. Discrete Math. 55 (1993), 81-88.

[11] A.M. Frieze and B.A. Reed, Polychromatic Hamiton cycles, Discrete Math. 118 (1993), 69-74.

[12] M.R. Garey and D.S. Johnson, Computers and Intractability, Freeman, New York (1976).

[13] G. Giraud, Sur les proportions respectives de triangles uni, bi ou tricolores dans un tricoloiage des arêtes du n-emble, Discrete Math. 16 (1976), 13-28.

[14] G. Hahn and C. Thomassen, Path and cycle sub-Ramsey numbers and an edgecoloring conjecture, Discrete Math. 62 (1986), 29-33.

[15] E.L. Lawler, Combinatorial Optimization: Networks and Matroids, Holt, Rinehart and Winston, New York (1976).

[16] Y. Manoussakis, M. Spyratos and Zs. Tuza, Cycles of given color patterns, J. Graph Theory 21 (1996), 153-162.

[17] Y. Manoussakis, M. Spyratos, Zs. Tuza and M. Voigt, Minimal colorings for properly colored subgraphs, Graphs and Combin. 12 (1996), 345-360.

[18] J.C. Picard and M. Queyranne, A network flow solution to some non-linear 0-1 programming problems, with applications to graph theory, Networks 12 (1982), $141-159$. 
[19] V. Rödl and Zs. Tuza, Rainbow subgraphs in properly edge-colored graphs, Random Structures and Algorithms 3 (1992), 175-182.

(Received 4 July 2003) 